\title{
A Note on a Maximum Principle for the DuFort-Frankel Difference Equation
}

\author{
By Paul Gordon
}

Consider the parabolic partial differential equation

$$
\partial u / \partial t=\sigma \partial^{2} u / \partial x^{2}
$$

where $\sigma$ is a positive constant.

Suppose initial and boundary conditions are given as follows:

$$
\begin{aligned}
u(0, x)=f_{1}(x): & & 0 \leqq x \leqq x_{1}, \\
u(t, 0)=f_{2}(t): & & 0 \leqq t \leqq t_{1}, \\
u\left(t, x_{1}\right)=f_{3}(t): & & 0 \leqq t \leqq t_{1} .
\end{aligned}
$$

Suppose that in the region $0 \leqq t \leqq t_{1}, 0 \leqq x \leqq x_{1}$, this data determines a continuously differentiable solution, $u(t, x)$, of Eq. (1). Let

$$
m=\max _{x, t}\left[\left|f_{1}(x)\right|,\left|f_{2}(t)\right|,\left|f_{3}(t)\right|\right] .
$$

It is well known that $u(t, x)$ satisfies the following boundedness property:

$$
|u(t, x)| \leqq m
$$

A difference equation representation for Eq. (1) would be expected, if it is to be convergent, to satisfy some kind of a bound similar to Eq. (3). The usual explicit and implicit difference equations satisfy precisely this bound [3, p. 13 and p. 47]. It is also well known that the DuFort-Frankel scheme satisfies some kind of a maximum principle. If one works with the $L_{2}$-norm, the form of the bound is quite clear [3, p. 83]. With respect to the maximum norm, it is also known that a maximum principle holds [2, p. 127], but its form is somewhat obscure. The purpose of this note is to derive the maximum principle satisfied by the DuFort-Frankel scheme in a relatively elementary fashion and to exhibit the dependence of this bound on the initial data.

The DuFort-Frankel difference equation can be written as follows:

$$
(1+q) U_{j}^{n+1}=(1-q) U_{j}^{n-1}+q\left(U_{j+1}^{n}+U_{j-1}^{n}\right),
$$

where

$$
q=2 \sigma \Delta t / \Delta x^{2}, \quad U_{j}^{n}=U(n \Delta t, j \Delta x) .
$$

Let us suppose that $\Delta x$ is specified as some function of $\Delta t, \Delta x=\Delta x(\Delta t)$. The consistency condition [3, p. 83$]$ requires

$$
\lim _{\Delta t=0}(\Delta t / \Delta x)=0 .
$$

Received September 21, 1967. 
Instead of proceeding in the time direction, the trick we employ is to suppose that the calculations proceed along the diagonals $x+t=$ constant. That is, at the $N$ th step obtain the values of $U_{j}{ }^{n}$ satisfying $n+i=N+2$. This means that at the $N$ th step the following system of equations is to be solved:

$$
(1+q) U_{i}^{N+2-i}-q U_{i+1}^{N+1-i}=(1-q) U_{i}^{N-i}+q U_{i-1}^{N+1-i}, \quad 1 \leqq i \leqq N .
$$

(If any of the other boundaries are encountered by the diagonal, the system of equations is simply cut off appropriately.) It is assumed that $U_{j}{ }^{0}, U_{j}{ }^{1}, U_{0}^{n}, U_{x_{1}}^{n}$ are known from the data, Eq. (2), and that the same bound is satisfied.

$$
m=\max _{x, t}\left[\left|U_{j}{ }^{0}\right|,\left|U_{j}{ }^{1}\right|,\left|U_{0}{ }^{n}\right|,\left|U_{x_{1}}^{n}\right|\right] .
$$

Let

$$
L_{j}^{n}=(1-q) U_{j}^{n}+q U_{j-1}^{n+1} .
$$

Then Eq. (7) can be solved as follows:

$$
(1+q) U_{N+2-i}^{i}=q\left(\frac{q}{1+q}\right)^{i-2} U_{N+1}^{1}+\sum_{\nu=0}^{i-2}\left(\frac{q}{1+q}\right)^{i-2-\nu} L_{N-\nu}^{\nu} .
$$

Let

$$
\bar{L}_{n}=\max _{\nu}\left\{\left|U_{n+1}^{1}\right|,\left|L_{n-\nu}^{\nu}\right|\right\} .
$$

Then,

$$
\left|U_{N+2-i}^{i}\right| \leqq \bar{L}_{N} .
$$

It remains to obtain a bound for $\bar{L}_{N}$. From Eqs. (9) and (10), after some manipulation, we obtain the following:

$$
\begin{aligned}
L_{n-i}^{i}= & \frac{1}{q}\left(\frac{q}{1+q}\right)^{i} U_{n-1}^{1}+\frac{1}{q^{2}}\left(\frac{q}{1+q}\right)^{i} \sum_{\nu=0}^{i-2}\left(\frac{1+q}{q}\right)^{v} L_{n-2-\nu}^{\nu} \\
& +\left(\frac{q}{1+q}\right) L_{n-1-i}^{i-1} .
\end{aligned}
$$

Thus,

$$
\bar{L}_{N} \leqq \max \left[\bar{L}_{N-2},\left|L_{N}{ }^{0}\right|,\left|L_{N-1}^{1}\right|,\left|U_{N+1}^{1}\right|\right] .
$$

But $L_{N}{ }^{0}$ and $L_{N}{ }^{1}$ depend on the initial data. A simple series expansion shows that

$$
\left|L_{N}{ }^{0}\right| \leqq C(\Delta t / \Delta x)+\left|U_{N}{ }^{0}\right|,
$$

where $C$ is determined by the data. The same holds for $L_{N}{ }^{1}$. Thus,

$$
\left|U_{j}{ }^{n}\right| \leqq m+C \frac{\Delta t}{\Delta x},
$$

where $\Delta t / \Delta x$ satisfies Eq. (6). Equation (15) is now to be compared with Eq. (4). 
General Electric Company

Space Sciences Laboratory

Valley Forge Space Technology Center

P. O. Box 8555

Philadelphia, Pennsylvania 19101

1. E. C. DuFort \& S. P. Frankel, "Stability conditions in the numerical treatment of parabolic differential equations," $M T A C$, v. 7, 1953, pp. 135-152. MR 15, 474.

2. G. E. Forsythe \& W. R. Wasow, Finite Difference Methods for Partial Differential Equations, Wiley, New York, 1960. MR 23 \#B3156.

3. R. D. Richtmyer, Difference Methods for Initial Value Problems, Interscience Tracts in Pure and Appl. Math, 4, Interscience, New York, 1957. MR 20 \#438. 